\section{Ein sicherer Thermostat unter Benutzung von Leuchtgas.}

Von W. P. JoRISSEN.

(Eingeg. 8./9. 1918.)

Bekanntlich wird gewöhnlich bei Thermostaten die gewählte Temperatur aufrecht erhalten durch automatisch regulierte Leuchtgasflämmchen. Läßt man diese auch in der Nacht brennen, so ist bisweilen viel, jedenfalls aber einige Gefahr für Brand vorhanden.

In den Figuren $1 a$ und $l b$ ist nun ein $g$ a $n z$ s i c h e r e r Thermostat mit Zubehör abgebildet.

Der Thermostat selber, ein Gefäß von Kupfer oder Zink, ist in Fig. $1 b$ rechts gezeichnet. Dieser enthält einen Temperaturregulator (links), einen Rührer (welcher gedreht wird von einem kleinen Heißluftmotor) und eine Bleiröhre zur eventuellen Durchströmung von kaltem Wasser. In der Wand sind zwei gläserne Fenster eingekittet, von welchen eins gezeichnet ist. Der Rest der Wand ist bekleidet von Asbestpappe.

Der Gebrauch von Kautschuk schläuchen is t vollständig ver $\mathrm{mi}$ i den. Statt dieser werden Bleiröhren mit verschiedenem Durchmesser benutzt, welche aneinander gelötet worden sind. Mit den Glasröhren des Thermoregulators (Fig. 1b) und mit dem

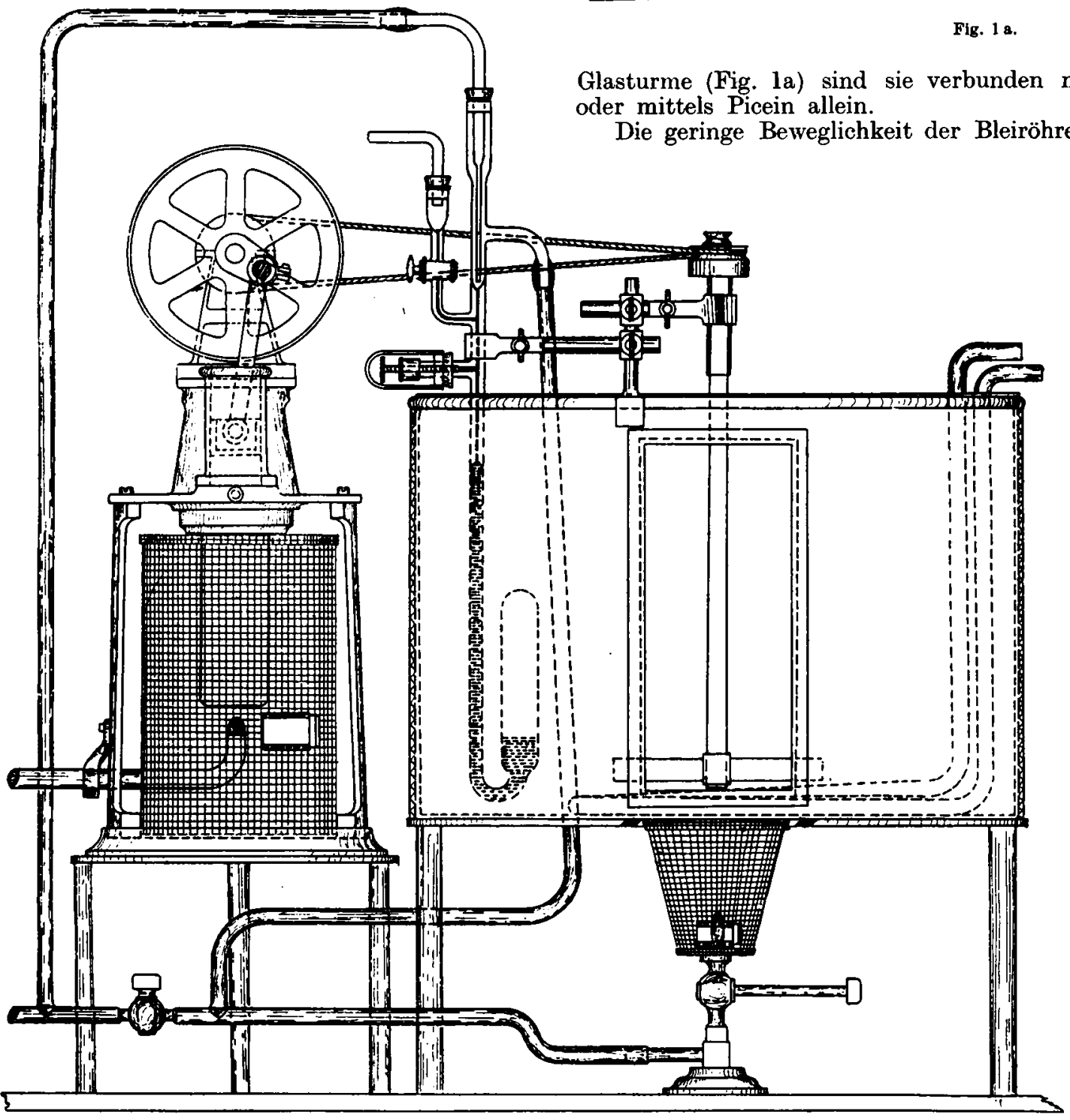

Fig. $1 b$

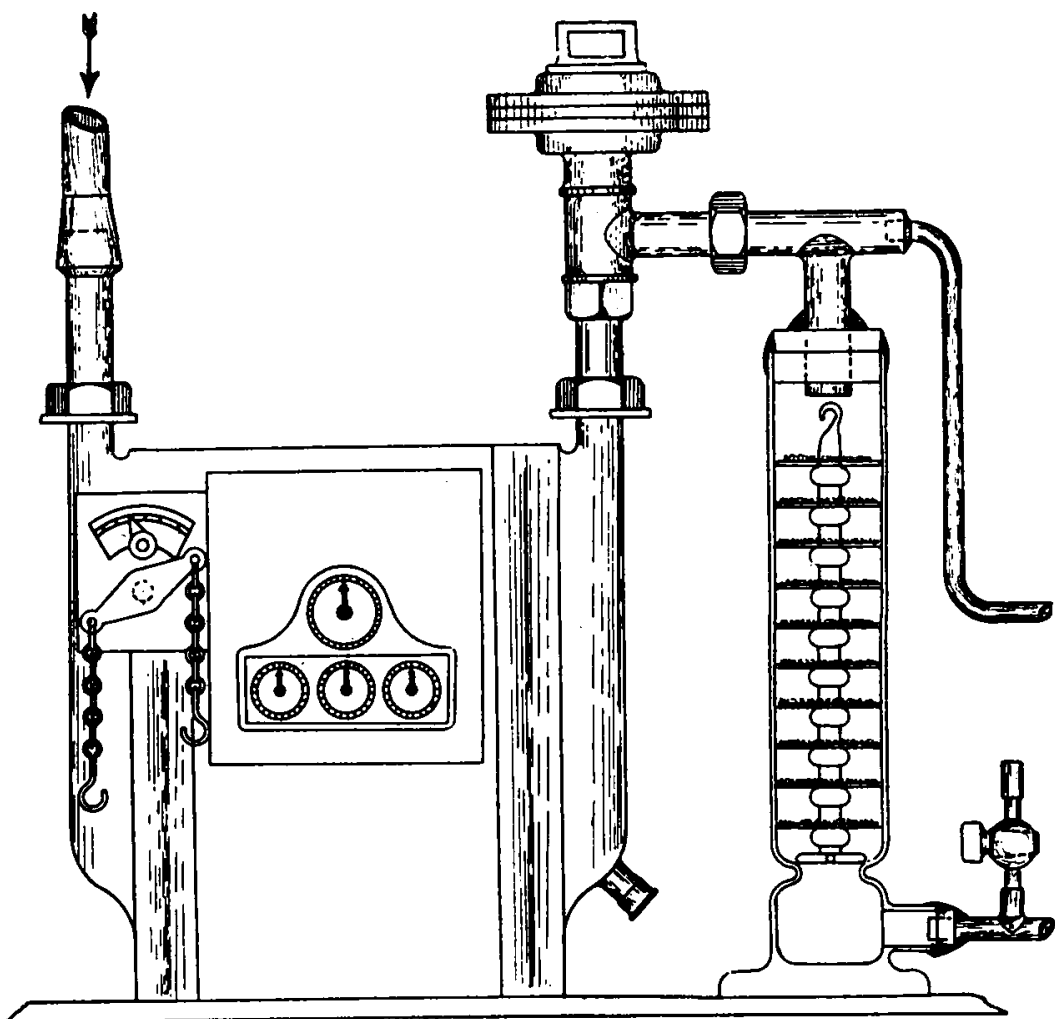

Fig. 1 .

(a) sind sie verbunden mittels Kork und Picein ${ }^{\mathbf{1}}$ )

Die geringe Beweglichkeit der Bleiröhren erlaubt nicht, die Temperatur einzustellen durch Heben oder Senken des engen Glasröhrchens im Os t w a ld schen Toluolregulator. Deshalb ist eine modifizierte Form ${ }^{2}$ ) ge wählt worden mit Einstellhahn zum bequemen Einfüllen und Absaugen des Quecksilbers beim Einstellen der Temperatur und mit einer Stahlfeinstellschraube zum genauen Einstellen des Meniscus. Die

Feinstellschraube kann nach dem Einstellen der Temperatur von einem Glasröhrchen beschützt werden. Die Flämmchen unter dem Thermostat und unter dem Heißluftmotor sind vollständig beschirmt von einem Messingdrahtnetz,

1) Eine schwarze Substanz, welche in Stangen, wie Siegellack, verkauft wird, von der „New York-Hamburger Gummiwaren-Compagnie " zu Hamburg. Sie haftet an Glas, Me tall, Holz usw., besonders wenn man die Gegenstände zuvor ein wenig erwärmt. Sie ist nicht spröde. Siehe auch: B. W a l te r, Ann. d. Phys. [4] 18, 860 (1905).

2) $\mathrm{Ost}$ wa ld - L u t h e r, Hand- und Hilfsbuch zur Ausführ. physiko-chem. Messungen. 2. Aufl. Auch: Hauptkatalog (B) (1905) von Fritz K ö hle r, Leipzig, S. 70, Fig. c. 
welches ungefähr 200 Öffnungen pro Quadratzentimeter besitzt, also zweimal soviel als $D$ a $v$ y für seine Bergwerklampe empfiehlt. Beim Flämmchen unter dem Thermostaten drückt das Drahtnetz an den Boden desselben, so daß es nicht überhitzt werden kann. Hier und beim Motor hat man in der Drahtnetzumhüllung eine Öffnung zum Anzünden der Flämmchen, welche jedoch nachher mittels eines genau schließenden Schubtürchens abgeschlossen wird.

Die Sicherheitsanordnung. wird nun vervollständigt durch Benutzung eines Sicherheitsgasmessers nach $\mathbf{J} . \mathbf{R} \mathbf{u} \mathbf{t}$ t e $n^{3}$ ) (siehe Fig. la). Er wird anstatt eines gewöhnlichen Gasmessers benutzt. In der Nähe der Gaseintrittsstelle (Fig. 1a) beobachtet man einen Heber mit zwei Ketten, hinter welchen man sich die Wörter ,Tag" und "Nacht" angestrichen denken muß. Zieht man den Heber auf „Nacht", so kann nur eine bestimmte Menge Gas, welche man eingestellt hat mittels des oberhalb des Hebers sich befindenden Zeigers, den Gasmesser während der Nacht passieren. Ist diese Menge verbraucht, so schließt der Gasmesser sich automatisch.

Wenn also ein Leck entsteht, so kann nur die bestimmte Menge Gas ausströmen, welche genügend war für etwaige Benutzung während der Nacht, aber ungenügend, um Gefahr für Explosion oder Erstickung (bei Benutzung in Wohnhäusern ${ }^{4}$ ) zu verursachen.

Beim Gebrauch im Laboratorium bestimmt man zuvor, wie viel Gas die Flämmchen verbrauchen während der Stunden, in welchen die Anordnung ohne Aufsicht ist. Man stellt den Zeiger auf die nächste höhere Zahl ein und zieht den Heber auf "Nacht".

Uber die Wirkungsweise eines der Modelle des $R u$ t t e $n$ schen Sicherheitsgasmessers ${ }^{5}$ ) gibt Fig. 2 Auskunft. Zieht man den Heber 7 auf ,Nacht", so dreht sich die exzentrische

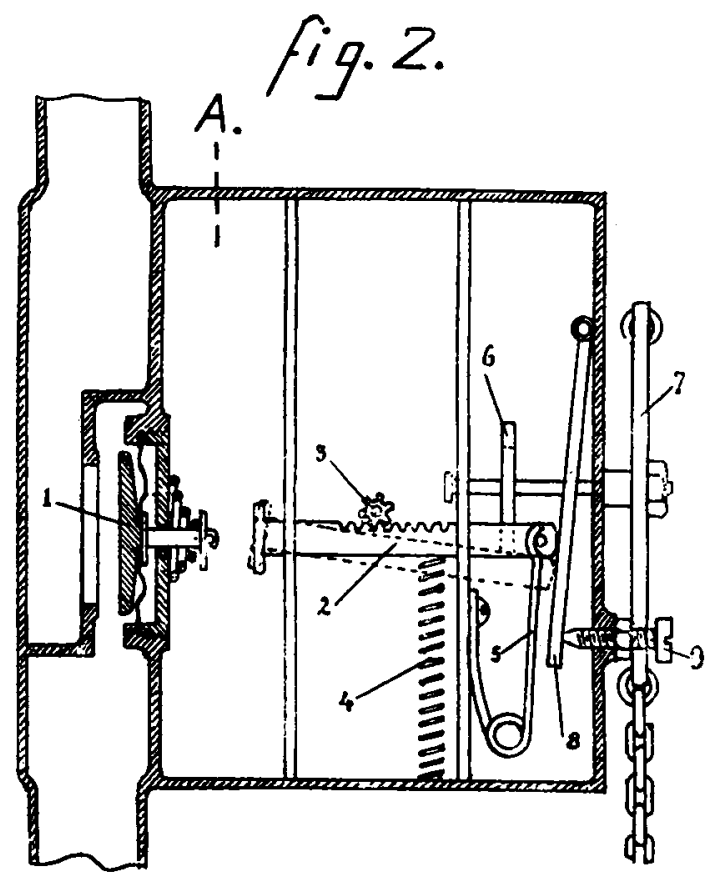

Scheibe 6 um ihre Achse, und es kann sich die Zahnstange 2, welche von der Feder 4 angepreßt wird, nach oben bewegen. Sie wird dann vom Zahnrad 3 (welches mit dem gewöhnlichen Mechanismus des Gasmessers in Verbindung steht) gegriffen und nach links bewegt, wobei sie die Feder 5 mitführt. Hat die Zahnstange 2 einen bestimmten Weg zurückgelegt, welcher von der Einstellplatte 8 bestimmt ist, so schließt sie bei 1 die Gaszufuhr allmählich ab. Zieht man

3) Chem. Ing., Adj. Dir. der Leuchtgasfabriken im Haag (Holland).

4) Für welche der Sicherheitsgasmesser eigentlich konstruiert worden ist.

5) Durch Patente geschützt. den Heber wieder auf "Tag", so drückt die exzentrische Scheibe 6 die Zahnstange 2 weg vom Rad 3, während die Feder 5 sie nach rechts bewegt, bis sie gehemmt wird von der Einstellplatte 8. Die Menge des für die Nacht verfüg. baren Gases wird eingestellt mittels der Schraube 9. Bei anderen Modellen, wie z. B. bei dem in Fig. la gezeichneten Messer, wird die Menge des Nachtgases auf andere Weise eingestellt.

Das Sicherheitsventil, das man in Fig. la rechts oben auf dem Gasmesser erblickt, ist in Fig. 3 gezeichnet. Wenn kein Druck in der Gasleitung ist (d. $h$. kein Druck ü b e r

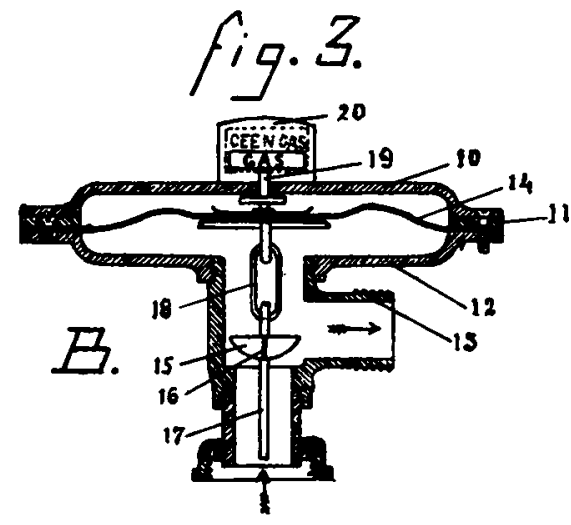

1 Atmosphäre), so ist die Membran 14 gesenkt, und es ruht die Klappe 15 auf der Öffnung der vertikalen Röhre. Zieht man nun den Heber auf ,Tag", und bekommt man also Druck unterhalb der Klappe 15, so kann das Gas durch die Spalte 16 und die Röhre 13 in die Leitung treten bis zu einem Maximum von $20 \mathrm{l}$ pro Stunde. Wenn nirgends in der Leitung ein Hahn geöffnet ist, und auch kein Leck von einiger Bedeutung besteht, so entsteht ein Druck (Utberdruck) in der Leitung, und also auch unterhalb der Membran 11. Ist der Druck (Uberdruck) gestiegen bis $15 \mathrm{~mm}$ Wasser, so wird die Klappe 15 aufgehoben, und kann vollständige Gaszufuhr stattfinden.

Falls während des ,Nacht"standes des Hebers alles verfügbare Gas verbraucht worden ist, so wird die Klappe 15 auf der Röhre ruhen, weil kein Utberdruck in der Leitung besteht. Zieht man nun den Heber auf "Tag", so wird, falls alle Hähne in der Leitung geschlossen sind oder nur sehr wenig Gas durchlassen, die Klappe 15 sofort sich erheben und das Wort ,Gas" erscheint an der Stelle 20. Ist dagegen ein Hahn offen geblieben, oder besteht ein Leck von einiger Bedeutung, so erhebt sich die Klappe nicht. Auch wenn am Tage ein so großes Leck entsteht, daß der Druck (Uberdruck) in der Leitung unter $15 \mathrm{~mm}$ Wasser sinkt, wird selbstverständlich die Gaszufuhr sofort automatisch abgeschlossen. Zum Schluß möge noch eine Vorrichtung beschrieben werden, welche auch in Fig. la rechts gezeichnet ist. Sie besteht aus einem Glasturm, in welchem eine Anzahl Scheiben Kupferdrahtnetz an einem Glasstabe befestigt worden sind. Auf diese Drahtnetze und auf dem Boden des Turmes ist Eisenoxydhydrat (Raseneisenerz) und mit Alkali behandelte Cellulose (Baumwolle oder Sägemehl) gelegt. Erstgenannte Substanz dient zur Beseitigung von noch vielleicht im Leuchtgase anwesenden Spuren Schwefelwasserstoff; letztgenannter nimmt den Schwefelkohlenstoff auf (der etwa $80 \%$ der im gereinigten Leuchtgase vorkommenden Schwefelverbindungen bildet).

Die Spuren Schwefelwasserstoff würden sonst den Quecksilbermeniscus im Regulator angreifen und so allmählich einen Einfluß auf die Temperaturregulierung ausüben können. Die immer anwesenden anderen Schwefelverbindungen bilden bei der Verbrennung Schwefelsäure, welche das Drahtnetz, die Wand des Thermostates usw. angreifen würde.

\section{L e i d e n (Holland), August 1913.}

Anorganisch-chemisches Laboratorium der Universitüt. 